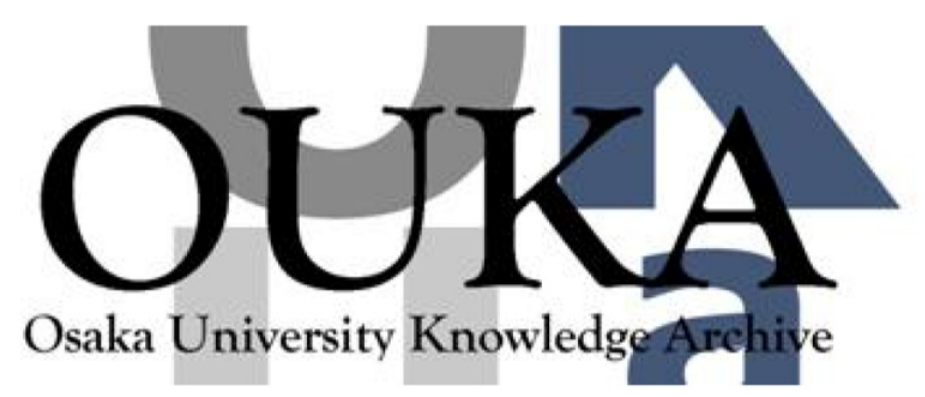

\begin{tabular}{|c|l|}
\hline Title & $\begin{array}{l}\text { COMPLEX METHODS IN HIGHER DIMENSIONS : RECENT } \\
\text { TRENDS FOR SOLVING BOUNDARY VALUE AND INITIAL } \\
\text { VALUE PROBLEMS }\end{array}$ \\
\hline Author(s) & Le, Hung Son; Tutschke, W. \\
Citation & $\begin{array}{l}\text { Annual Report of FY 2005, The Core University } \\
\text { Program between Japan Society for the Promotion } \\
\text { of Science (JSPS) and Vietnamese Academy of } \\
\text { Science and Technology (VAST). p. 323-p. 331 }\end{array}$ \\
\hline Issue Date & 2006 \\
\hline oaire:version & VoR \\
\hline URL & https://hdl. handle. net/11094/12972 \\
\hline rights & \\
\hline Note & \\
\hline
\end{tabular}

Osaka University Knowledge Archive : OUKA

https://ir. Library. osaka-u. ac. jp/

Osaka University 


\title{
COMPLEX METHODS IN HIGHER DIMENSIONS \\ - RECENT TRENDS FOR SOLVING BOUNDARY VALUE AND INITIAL VALUE PROBLEMS
}

\author{
Le Hung Son
}

Hanoi University of Technology, Faculty of Applied Mathematics and Informatics Dai Co Viet Road 1, 10000 Hanoi, Vietnam, e-mail: lehung@netnam.org.vn

$$
\text { W. TUTSCHKE }
$$

Graz University of Technology, Department of Mathematics D Steyrergasse 30/3, A-8010 Graz, Austria, e-mail: tutschke@tugraz.at

\begin{abstract}
From the very beginning, Complex Analysis is closely connected with partial differential equations. The paper surveys present generalizations of these connexions to higher dimensions.
\end{abstract}

\section{Boundary and initial value problems in classical Complex Analysis}

The two basic connexions between Complex Analysis and partial differential equations are the Cauchy-Riemann system for real and imaginary part of a holomorphic function $w=u+i v$ and the Cauchy-Kovalevskaya Theorem. The first fact shows that on can find holomorphic functions with arbitrary 
boundary values of the real part (since $\Delta u=0$ ). Knowing $u$, the imaginary part is uniquely determined up to an arbitrary real constant.

Boundary value problems can also be solved not only for the linear Vekua equation

$$
\frac{\partial w}{\partial \bar{z}}=a(z) w+b(z) \bar{w}
$$

(see I. N. Vekua's monograph [?]), but also for the fully non-linear complex first order equation

$$
\frac{\partial w}{\partial \bar{z}}=\mathcal{F}\left(z, w, \frac{\partial w}{\partial z}\right)
$$

(see [?]).

The second connexion between Complex Analysis and partial differential equations, the Cauchy-Kovalevskaya Theorem, can also be extended to generalizations of the Cauchy-Riemann system. Under suitable conditions, an initial value problem of Cauchy-Kovalevskaya type can be constructed for initial functions which are solutions of an equation of the form (1) (see [?]).

\section{Complex methods in higher dimensions}

Of course, boundary and initial value problems in higher dimensions can be solved also in the framework of Real Analysis. Methods of Complex Analysis, however, lead to additional information at least for some classes of equations.

Generally speaking, there are three possibilies to apply complex methods in higher dimensions:

a) One considers a higher-dimensional system of differential equations having similar properties as the Cauchy-Riemann system has. Using arguments of classical Complex Analysis, one tries to prove similar statements such as a generalized Cauchy Integral Formula and a generalized Cauchy-Pompeiu Formula. Such a higher-dimensional analog to the Cauchy-Riemann system is, for instance, the system

$$
\begin{aligned}
& \operatorname{div} u=0 \\
& \text { curl } u=0
\end{aligned}
$$


for so-called potential vectors $u=\left(u_{1}, u_{2}, u_{3}\right)$ in $\mathbf{R}^{3}$.

b) Another possibility for using ideas of Complex analysis in higher dimensions is to investigate complex-valued functions in several complex variables.

c) A third possibility, finally, is to investigate equations containing the Cauchy-Riemann operator

$$
D=\sum_{j=0}^{n} e_{j} \frac{\partial}{\partial x_{j}}
$$

of Clifford Analysis where the desired functions depend on $x_{0}, x_{1}, \ldots, x_{n}$ and are Clifford algebra valued. Then the so-called (left-)monogenic functions $u_{0}$ satisfying the generalized Cauchy-Riemann equation $D u_{0}=0$ can be interpreted as holomorphic functions in $\mathbf{R}^{\mathbf{n + 1}}$.

\section{Boundary value problems}

Consider a boundary value problem of the form

$$
\begin{aligned}
& \mathcal{L} u=F(x, u) \text { in } \Omega \\
& \mathcal{B} u=g \text { on } \partial \Omega
\end{aligned}
$$

where $\mathcal{B}$ is an operator acting on the boundary $\partial \Omega$ of a domain $\Omega$ in $\mathbf{R}^{\mathbf{n}}$. The simplest examples for such boundary operators are the restriction of the desired solution $u$ to the boundary (Dirichlet problem) or to prescribe the normal derivative (Neumann problem). In case $\mathcal{L}$ is a linear operator possessing a fundamental solution $E(x, \xi)$, fixed points of the operator

$$
U(x)=u_{0}+\int_{\Omega} E(x, \xi) F(\xi, u(\xi)) d \xi,
$$

where $u_{0}$ is a solution of the simplified equation $\mathcal{L} u_{0}=0$, turn out to be solutions of the (non-linear) differential equation (3) (cf. [?]). In addition, the boundary condition (4) is satisfied if the boundary values of $u_{0}$ are given by the condition $\mathcal{B U}=g$ on $\partial \Omega$. In this way boundary value problems for equations of the form (3) can be reduced to boundary value problems for the simplified equation $\mathcal{L} u_{0}=0$. 
Since

$$
-\frac{1}{\pi} \cdot \frac{1}{\zeta-z}
$$

is a fundamental solution of the Cauchy-Riemann system, boundary value problems for the non-linear system (2) can be reduced to boundary value problems for holomorphic functions. Using the fundamental solution of the Cauchy-Riemann equation $D u=0$ in $\mathbf{R}^{\mathbf{n + 1}}$, in a similar way boundary value problems for

$$
D u=F(x, u)
$$

can be reduced to boundary value problems for monogenic functions.

Concerning boundary value problems for monogenic functions (and thus also for solutions of systems of the form (5)), one can solve the Dirichlet boundary value problem for one component of the monogenic function (because each component satisfies the Laplace equation). The other components can be prescribed only on a lower-dimensional part of the boundary (see, for instance, the paper [?] of I. Stern), according to the fact that the imaginary part of a holomorphic function is determined up to a (real) constant if one prescribes the real part on the boundary.

Research to be done in the framework of the above fixed-point method should also include a comparison of different norms (sup-norm, Hölder norm, $L_{p^{-}}$ norm). Generally speaking, fixed-point method are to be applied in closed subsets such as balls of the underlying function space. Better results can thus be obtained in optimal balls or optimal polycylinders (see [?] and S. Graubner $[4,5,6]$ resp.).

An introduction into boundary value problems for functions in several complex variables can be found in H. G. W. Begehr's and A. Dzhuraev's book [2]

\section{Initial value problems}

Solutions $u=u(t, x)$ of the initial value problem

$$
\begin{aligned}
& \partial_{t} u=\mathcal{F}\left(t, x, u, \partial_{x_{j}} u\right) \\
& u(0, x)=\varphi(x)
\end{aligned}
$$


where $t$ means the time and $x=\left(x_{1}, \ldots, x_{n}\right)$ is a point of $\mathbf{R}^{\mathbf{n}}$ are fixed points of the integro-differential operator

$$
U(t, x)=\varphi(x)+\int_{0}^{t} \mathcal{F}\left(\tau, x, u, \partial_{x_{j}} u\right) d \tau .
$$

Fixed points of (8) can be constructed by the contraction-mapping principle under the following assumptions:

a) $\mathcal{F} u$ satisfies the (global) Lipschitz condition

$$
\|\mathcal{F} u-\mathcal{F} v\| \leq L_{0}\|u-v\|+\sum_{j} L_{j}\left\|\partial_{x_{j}} u-\partial_{x_{j}} v\right\|
$$

with respect to the norm of a certain Banach space $\mathcal{B}$ with respect to the spacelike variables (where $x$ runs in a domain $\Omega$ of $\mathbf{R}^{\mathrm{n}}$ ).

b) The operator $\mathcal{F}$ on the right-hand side of (8) possesses an associated operator $\mathcal{G}$ (with time-independent coefficients), that is, $\mathcal{G} u=0$ implies $\mathcal{G}(\mathcal{F} u)=0($ for each fixed $t)$.

c) The solutions of $\mathcal{G} u=0$ satisfy an interior estimate of the form

$$
\left\|\partial_{x_{j}} u\right\|_{\mathcal{B}\left(\Omega^{\prime}\right)} \leq \frac{\text { const }}{\operatorname{dist}\left(\Omega^{\prime}, \partial \Omega^{\prime \prime}\right)}\|u\|_{\mathcal{B}\left(\Omega^{\prime \prime}\right)}
$$

where $\Omega^{\prime}$ is a subdomain of $\Omega^{\prime \prime}$.

In order to estimate the integral operator (8), one exhausts the given domain $\Omega$ by a family of subdomains $\Omega_{s}, 0<s<s_{0}$. Assign to each $x$ a (uniquely determined) index $s(x)$ such that $x$ belongs to the boundary of $\Omega_{s(x)}$. Then one can contruct the conical domain

$$
M=\left\{(t, x): x \in \Omega, 0 \leq t<\eta\left(s_{0}-s(x)\right)\right\}
$$

in the $t, x$-space and height equals $\eta s_{0}$, where $\eta$ will be fixed later. Using the interior estimate mentioned above, one can show that the operator is contractive provided $\eta$ is small enough. Applying the contraction-mapping principle, one has thus proved that the initial value problem (6), (7) is locally solvable. The initial function $\varphi$ has to satisfy the associated equation $\mathcal{G} \varphi=$ 0 . On the other hand, the constructed solution $u(t, x)$ satisfies also the associated equation for each $t$. 
The above approach to the initial value problem (6), (7) (see [?]) generalizes a construction given by W. Walter in his paper [?].

Interior estimates can be found by boundary integral representations and are for different norms possible in the case of elliptic equations, in general. Concerning associated pairs, two types of problems can be solved. Either $\mathcal{F}$ is given, and one has to determine an associated equation $\mathcal{G} u=0$ in which the initial value problem is solvable. Or $\mathcal{G}$ is given, and one determines all $c F$ for which solutions $\varphi$ of $\mathcal{G} \varphi=0$ are admissible initial functions.

If $\mathcal{F}$ is given, it can happen that there exist several associated equations. In some cases it is then even possible to solve ill-posed initial value problems (see [?]).

Sufficient conditions for associated pairs can be obtained by comparison of coefficients (after one has eliminated superfluous derivatives using the associated equation $\mathcal{G} u=0$ ). Substituting special solutions, one can sometimes show that the obtained conditions are also necessary. In the paper [7], for instance, all linear first order systems for two desired real-valued functions are determined which are associated to the Cauchy-Riemann system.

In the case of the Cauchy-Riemann system the necessary interior estimate follows from Cauchy's Integral Formula. Analogously, for monogenic functions the interior estimate can be obtained from the Poisson Integral Formula for harmonic functions because monogenic functions are solutions of the Laplace equation.

The construction of associated pairs in the framework of Clifford analysis will by illustrated by the next section.

\section{An example. Associated pairs in Clifford analysis}

Let $\Omega$ ba a domain in $\mathbf{R}^{3}$, and let $\mathcal{G}$ be the generalized Cauchy-Riemann operator in $\mathrm{R}^{3}$, that is,

$$
\mathcal{G} u=\frac{\partial u}{\partial x_{0}}+e_{1} \frac{\partial u}{\partial x_{1}}+e_{2} \frac{\partial u}{\partial x_{2}}
$$


where $u=u_{0}+u_{1} e_{1}+u_{2} e_{2}+u_{12} e_{1} e_{2}$ is a Clifford algebra valued function. Consider operators of the form

$$
\mathcal{F} u \equiv A^{(0)}(t, x) \frac{\partial u}{\partial x_{0}}+A^{(1)}(t, x) \frac{\partial u}{\partial x_{1}}+A^{(2)}(t, x) \frac{\partial u}{\partial x_{2}}
$$

whose coefficients are Clifford algebra valued. This operator is associated to the Cauchy-Riemann operator if (for each fixed $t$ ) it transforms monogenic functions into monogenic functions, that is, $D u=0$ implies $D(\mathcal{F} u)=0$. The equation $D u=0$ implies

$$
\frac{\partial u}{\partial x_{0}}=-e_{1} \frac{\partial u}{\partial x_{1}}-e_{2} \frac{\partial u}{\partial x_{2}} .
$$

Similarly, one can express the mixed second derivatives $\partial^{2} u / \partial x_{0} \partial x_{1}$ and $\partial^{2} u / \partial x_{0} \partial x_{2}$ by $\partial^{2} u / \partial x_{1}^{2}, \partial^{2} u / \partial x_{1} \partial x_{2}$ and $\partial^{2} u / \partial x_{2}^{2}$. Taking into account these relations, it follows that $D(\mathcal{F} u)$ can be expressed as a linear combination of

$$
\frac{\partial u}{\partial x_{1}}, \frac{\partial u}{\partial x_{2}}, \frac{\partial^{2} u}{\partial x_{1}^{2}}, \frac{\partial^{2} u}{\partial x_{1} \partial x_{2}} \text { and } \frac{\partial^{2} u}{\partial x_{2}^{2}} .
$$

Equating its coefficients to zero, one gets a sufficient condition for $\mathcal{F}$ to be associated to the Cauchy-Riemann operator. The 2 coefficients of the first order derivatives of $u$ lead to the two equations

$$
\begin{aligned}
& D A^{(1)}=D A^{(0)} \cdot e_{1} \\
& D A^{(2)}=D A^{(0)} \cdot e_{2} .
\end{aligned}
$$

Rewriting $A^{(k)}$ componentwise,

$$
A^{(k)}=A_{0}^{(k)}+A_{1}^{(k)} e_{1}+A_{2}^{(k)} e_{2}+A_{12}^{(k)} e_{1} e_{2}, \quad k=0,1,2,
$$

the 3 coefficients of the second order derivatives show that the $A_{j}^{(k)}(k=$ $0,1,2, j=0,1,2,12)$ have to satisfy 5 relations:

$$
\begin{aligned}
& A_{1}^{(1)}=A_{12}^{(0)}, A_{12}^{(1)}=-A_{2}^{(0)} \\
& A_{1}^{(2)}=A_{12}^{(0)}, A_{2}^{(2)}=A_{12}^{(0)}, A_{12}^{(2)}=A_{1}^{(0)} .
\end{aligned}
$$

So the 5 coefficients on the left-hand side of these equations can be eliminated from the equations (9), (10). Doing this, one obtains 8 homogeneous (linearly independent) equations for the first order derivatives of

$$
A_{0}^{(0)}, A_{1}^{(0)}, A_{2}^{(0)}, A_{12}^{(0)}, A_{0}^{(1)}, A_{2}^{(1)}, A_{0}^{(2)} .
$$


If these equations are satisfied, then $\mathcal{F}$ is associated to the generalized Cauchy-Riemann operator in $\mathrm{R}^{3}$. In order to construct admissible associent operators, we assume that the desired coefficients are linear functions in the spacelike variales $x_{0}, x_{1}$ and $x_{2}$. Then the 8 sufficient relations for associated pairs lead to a linear and homogeneous system for the coefficients of the above mentioned $A_{j}^{(k)}$. Since the rank of this system is equal to 8 , one finds $21-8=13$ linearly independent associated operators $\mathcal{F}$ whose coefficients are linear functions in the spacelike variables. Note, however, that the free coefficients can be chosen as arbitrary (continuous) functions in $t$.

If the right-hand side $\mathcal{F}$ of (6) and the generalized Cauchy-Riemann operator $D$ of Clifford analysis form an associated pair, then each monogenic function $\varphi$ is an admissible initial function (7).

\section{References}

[1] H.G.W. Begehr, A.O. Çelebi, and W. Tutschke (eds.), Complex Methods for Partial Differential Equations. Proceedings of the Workshop on "Recent Trends in Complex Methods for Partial Differential Equations", held at Middle East Technical University in Ankara, July 6-10, 1998. Kluwer Academic Publishers, ISAAC series, vol. 6, 1999.

[2] H. G. W. Begehr and A. Dzhuraev; ***

[3] H. Florian, N. Ortner, F. J. Schnitzer, and W. Tutschke (eds.), Functional-analytic and complex methods, their interactions, and applications to partial differential equations. World Scientific 2001.

[4] S. Graubner, On the optimization of polycylinders in applications of the contraction-mapping principle to differential equations (in German). Thesis (Dr. rer. nat. dissertation) at Free University Berlin, 1997.

[5] - On the optimization of the set of definition of operators in applications of the contraction-mapping principle to differential equations. Contained in the collection of papers [1], pp. 73-76.

[6] -, Optimization of fixed-point methods. Contained in the collection of papers [3], pp. 91-100. 
[7] Le Hung Son and W. Tutschke, First Order Differential Operators 99 